\title{
Optimizing Radar Waveforms Using Generalized Alternating Projections
}

\author{
Dylan Eustice, Charles Baylis, \\ Casey Latham, and Robert J. Marks II \\ Wireless and Microwave Circuits and Systems Program \\ Department of Electrical and Computer Engineering \\ Baylor University \\ Waco, TX, USA
}

\author{
Lawrence Cohen \\ Radar Division \\ United States Naval Research Laboratory \\ Washington, DC, USA
}

\begin{abstract}
The radar ambiguity function, described as an autocorrelation of shifts in time and frequency, is useful for determining a waveform's accuracy at detecting targets in certain range-Doppler combinations. An algorithm is proposed which uses a generalized method of alternating projections to synthesize waveforms with desired ambiguity function properties. In practice, it is often desirable to minimize the magnitude of the ambiguity function at range-Doppler combinations where targets other than the detection are likely to cause interference. The algorithm alternates between projections in the time, frequency, and range-Doppler domains until an optimal solution which fits desired ambiguity function properties is found. This work provides a computationally intelligent methodology to dynamically optimize detection in radar applications and a foundation for future work in joint circuit optimization for spectral compliance.
\end{abstract}

Keywords-ambiguity function, projection onto convex sets, waveform optimization

\section{INTRODUCTION}

Woodward's ambiguity function [1] is an autocorrelation which describes the ability of a waveform, $x(t)$, to precisely detect targets and is defined as

$$
\chi(\tau, u)=\int_{-\infty}^{\infty} x(t) x^{*}(t-\tau) e^{-j 2 \pi u t} d t
$$

where $\tau$ and $u$ are the range and Doppler errors relative to the range and Doppler of the detected target. The ideal ambiguity function is one with zero ambiguity at all range and Doppler with the exception of the origin, a shape which resembles a thumbtack. Unfortunately, it is not possible to physically realize a waveform which produces such an ambiguity function [2]. It is also important to note that the total volume of the ambiguity function is dependent on the energy of the signal, leading to necessary tradeoffs that must be made in waveform design.

There are generally two different approaches taken to address this problem. The first seeks to model the ambiguity function design after the thumbtack shape, producing high ambiguity near the origin and spreading the remainder of the volume evenly across the range-Doppler plane. This waveform design is useful for more general detection applications where there is little or no prior knowledge as to where other targets are located in the range-Doppler domain relative to the target detected. Costas [3] presents a signal class which is a good representation of this technique. The second approach attempts to minimize the ambiguity function in areas where other targets are likely to be relative to the detection, seeking to constrain the volume to other range-Doppler combinations. Extensive work has gone into synthesizing ambiguity functions with volume properties fitted to those of a desired function, as seen in [4-6].

The function defined in (1) is sometimes known as the auto-ambiguity function. An alternate definition, the crossambiguity function, is the autocorrelation between a pair of waveforms as opposed to the single waveform. Sussman [4], who presents a least-squares method of approximating an ambiguity function to a desired function, focuses on the crossambiguity function and treats the auto-ambiguity function as a special case. He et al. [5] also focus on the cross-ambiguity function and propose a waveform synthesis algorithm which optimizes a pair of waveforms simultaneously. In both $\mathrm{He}$ and Sussman's works, a catalog of signals is used as a set of basis functions which make up the pair of waveforms designed. One novel aspect of the present paper is that the algorithm produces an entirely new waveform, constructed without the use of any basis functions. Additionally, we focus on optimization of the auto-ambiguity function, which in theory is a more challenging problem due to the fewer degrees of freedom available relative to the cross-ambiguity function. However, there is no reason to assume the effectiveness of the methodology used is strictly limited to the auto-ambiguity function.

Projection onto convex sets (POCS) is a simple algorithm first introduced by Gubin et al. [7] whose applications extend to a broad range of areas [8]. The algorithm solves for the intersection or near point between two or more sets by alternating projections between sets. Convergence is only guaranteed if all of the sets are convex and their intersection is non-empty. POCS has been successfully applied in waveform design in the past. Civanlar et al. demonstrate POCS's usefulness in digital pulse shaping in [9], [10] and Tropp et al. use POCS to develop signature sequences with low peak-to-average power ratios in CDMA systems [11]. This paper uses an application of POCS to optimize the radar waveform for desired-ambiguity function properties. 


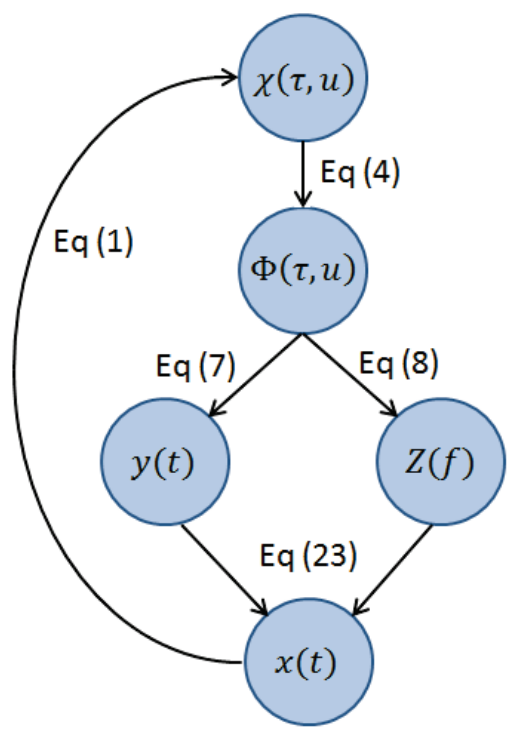

Fig. 1. Flowchart of the projections in the algorithm.

\section{Problem Formulation}

Assume that a two-dimensional minimization function, $M(\tau, u)$, has been designed which has zero magnitude in range-Doppler combinations where targets are likely to exist, relative to the target being detected, and arbitrary magnitude in regions where erroneous detection would not be harmful, such as regions where other targets are known not to be located relative to the detection. We seek to find a waveform $x(t)$ such that the magnitude of its ambiguity function, $\chi(\tau, u)$, is minimized in range-Doppler regions corresponding to those in $M(\tau, u)$. Given the set of minimized functions $M$ and the set of ambiguity functions $A$, the optimum is reached when the criteria

$$
\chi(\tau, u) \in A \cap M
$$

is met.

As previously noted, POCS convergence is only guaranteed if all sets are convex and their intersection is non-empty. Following the classic definition of convexity [12], a set of signals $R$ is only convex if

$$
\alpha u_{1} t+(1-\alpha) u_{2}(t) \in R
$$

where

$$
\begin{array}{r}
u_{1}(t), u_{2}(t) \in R \\
0 \leq \alpha \leq 1
\end{array}
$$

Under this definition, the set of ambiguity functions $A$ is not convex. As a result, convergence is not guaranteed when applying the classical POCS algorithm and we apply a modified version of POCS, also referred to as generalized POCS.

\section{Proposed Algorithm}

In this section, the steps of the proposed generalized POCS algorithm are examined. The performance of the algorithm as a whole and a comparison of its variations will be quantified in the following section.

\section{A. Overview of Projections}

A total of four projections are used: the projection of the ambiguity function onto the set of functions minimized at selected range-Doppler combinations $\chi(\tau, u) \rightarrow \Phi(\tau, u)$, the projections of the set of functions minimized at selected range-Doppler combinations onto the the time and frequency domains $\Phi(\tau, u) \rightarrow y(t), Z(f)$, and the projection of the time domain waveform onto its ambiguity function $x(t) \rightarrow \chi(\tau, u)$.

Given a set of range-Doppler combinations $B$ where $M(\tau, u)$ is minimized, we can project $\chi(\tau, u)$ onto $\Phi(\tau, u)$ by taking

$$
P_{M}(\chi(\tau, u))= \begin{cases}\min \{M(\tau, u), \chi(\tau, u)\}, & (\tau, u) \in B \\ \chi(\tau, u), & \text { otherwise }\end{cases}
$$

For the purposes of projecting the minimized function $\Phi(\tau, u)$ onto time and frequency signals, we treat $\Phi(\tau, u)$ as an ambiguity function. Note that in reality, $\Phi(\tau, u)$ will not be in the set of ambiguity functions unless the optimum has been reached. To derive the projections, we see that a time or frequency signal may be found in terms of its ambiguity function, to within a scaling factor. Recall (1), which is equivalent to,

$$
\chi(\tau, u)=\mathcal{F}\left\{x(t) x^{*}(t-\tau)\right\}
$$

taking the inverse Fourier

$$
\int_{-\infty}^{\infty} \chi(\tau, u) e^{j 2 \pi u t} d u=x(t) x^{*}(t-\tau)
$$

and setting $t=0$ and $\tau=-\tau$, we arrive at

$$
\left[\frac{1}{x(0)} \int_{-\infty}^{\infty} \chi(-\tau, u) d u\right]^{*}=x(\tau)
$$

Following a similar set of operations for the frequency definition of the ambiguity function,

$$
\chi(\tau, u)=\int_{-\infty}^{\infty} X^{*}(f) X(f+u) e^{j 2 \pi \tau f} d f
$$

We find

$$
\frac{1}{X^{*}(0)} \int_{-\infty}^{\infty} \chi(\tau, u) d \tau=X(u)
$$

Using (5) and (6) and disregarding the scaling factors $x^{*}(0)$ and $X^{*}(0)$, we write the projections as

$$
\begin{array}{ll}
P_{t}(\Phi(\tau, u)): & y(t)=\int_{-\infty}^{\infty} \Phi^{*}(-\tau, u) d u \\
P_{f}(\Phi(\tau, u)): & Z(f)=\int_{-\infty}^{\infty} \Phi(\tau, u) d \tau
\end{array}
$$

Finally, the projection of the waveform $x(t)$ onto $\chi(\tau, u)$ is done by applying (1). 


\section{B. Weighting Projections}

After projecting $\Phi(\tau, u)$ onto the time and frequency domains to yield signals $y(t)$ and $Z(f)$, respectively, the candidate waveform is found via a weighted linear combination of the two projections. The weighting is dependent on the quality of each projection, which is quantified with the function $q$. Two different definitions of $q$ are applied, depending on user input. Both definitions of $q$ are dependent on the magnitude of the difference in the range and Doppler projections from $\Phi(\tau, u)$ and $\chi(\tau, u)$.

Recall (7) and (8), where the projection onto the time domain was dependent on the integral along the Doppler domain and the projection onto the frequency domain was dependent of the integral along the range domain. A similar methodology is applied, which will rank the quality of the projections based on the amount of change in each projection's respective domain. Given a two-dimensional function $\beta(\tau, u)$, let

$$
\begin{aligned}
& A_{t}(\beta(\tau, u))=\int_{-\infty}^{\infty} \beta(\tau, u) d u \\
& A_{f}(\beta(\tau, u))=\int_{-\infty}^{\infty} \beta(\tau, u) d \tau
\end{aligned}
$$

Note that higher values of $q$ correspond to a worse projection, with an optimal projection being $q=0$.

The first definition of $q$ applies a minimax approach, using the largest difference in magnitude between the projection of $\Phi(\tau, u)$ and the projection of $\chi(\tau, u)$.

$$
\begin{gathered}
q_{t}(\Phi(\tau, u), \chi(\tau, u))= \\
\left.\max _{t} \mid A_{t}(\chi(\tau, u))-A_{t}(\Phi(\tau, u))\right) \mid \\
q_{f}(\Phi(\tau, u), \chi(\tau, u))= \\
\left.\max _{f} \mid A_{f}(\chi(\tau, u))-A_{f}(\Phi(\tau, u))\right) \mid
\end{gathered}
$$

The second definition of $q$ applies a least-squares approach, taking the squared sum of the difference in magnitude between projections.

$$
\begin{gathered}
q_{t}(\Phi(\tau, u), \chi(\tau, u))= \\
\int_{-\infty}^{\infty}\left|A_{t}(\chi(\tau, u))-A_{t}(\Phi(\tau, u))\right|^{2} d t \\
q_{f}(\Phi(\tau, u), \chi(\tau, u))= \\
\int_{-\infty}^{\infty}\left|A_{f}(\chi(\tau, u))-A_{f}(\Phi(\tau, u))\right|^{2} d f
\end{gathered}
$$

\section{Distance Function}

To determine the quality of the candidate waveform $x_{i}(t)$, we define a distance function $d$. $d$ is intended to measure how well $\chi(\tau, u)$ is fitted to $M(\tau, u)$, with two options available for its definition: a minimax definition and a leastsquares definition. The minimax definition finds the worst case range-Doppler ambiguity while the integral definition finds the quality of the fit across all range-Doppler combinations. Under both definitions, $d$ will be 0 when the criteria in (2) is met.
The minimax definition follows a process similar to that used in [13] and is equal to the value of the normalized maximum magnitude of $\chi(\tau, u)$ within the minimization region $B$, or

$$
d(\chi(\tau, u), M(\tau, u))=\max _{(\tau, u) \in B} \frac{|\chi(\tau, u)|}{|\chi(0,0)|}
$$

where $B$ is the set of range-Doppler combinations where $|\chi(\tau, u)|>|M(\tau, u)|$.

The least-squares approach of $d$ is the squared difference between the minimized function $\operatorname{Phi}(\tau, u)$ and $\chi(\tau, u)$

$$
\begin{aligned}
& d(\chi(\tau, u), M(\tau, u))= \\
& \int_{-\infty}^{\infty} \int_{-\infty}^{\infty}\left(\frac{|\chi(\tau, u)-\Phi(\tau, u)|}{\chi(0,0)}\right)^{2} d u d \tau
\end{aligned}
$$

\section{Outline}

The algorithm is a iterative process with eleven main steps. Let $P$ denote a projection and $i$ represent the iteration index.

1) Build the minimization function $M(\tau, u)$ and the initial ambiguity function $\chi_{0}(\tau, u)$

2) Project $\chi_{i}(\tau, u)$ onto the set of minimized functions

$$
\Phi_{i}(\tau, u)=P_{M}\left(\chi_{i}(\tau, u)\right)
$$

3) Find the distance quality of $\chi_{i}(\tau, u)$

$$
Q_{i-1}=d\left(\chi_{i}(\tau, u), M(\tau, u)\right)
$$

4) Project $\Phi_{i}(\tau, u)$ onto the time domain

$$
y_{i}(t)=P_{t}\left(\Phi_{i}(\tau, u)\right)
$$

5) Project $\Phi_{i}(\tau, u)$ onto the frequency domain

$$
Z_{i}(f)=P_{f}\left(\Phi_{i}(\tau, u)\right)
$$

6) Using a weighting function $q$, find the quality of both the time and frequency domain projections

$$
\begin{aligned}
& Q_{t}=q_{t}\left(\Phi_{i}(\tau, u), \chi_{i}(\tau, u)\right) \\
& Q_{f}=q_{f}\left(\Phi_{i}(\tau, u), \chi_{i}(\tau, u)\right)
\end{aligned}
$$

7) Find the weighted combination of the time and frequency projections to find a candidate signal $x_{i}(t)$

$$
x_{i}(t)=\frac{1}{Q_{t}} y_{i}(t)+\frac{1}{Q_{f}} \mathcal{F}^{-1}\left\{Z_{i}(f)\right\}
$$

8) Normalize the signal energy to a predetermined value $E$

$$
x_{i}(t)=x_{i}(t) \sqrt{\frac{E}{\int_{-\infty}^{\infty}\left|x_{i}(t)\right|^{2} d t}}
$$

9) Calculate the ambiguity function $\chi_{i+1}(\tau, u)$ produced by $x_{i}(t)$

$$
\chi_{i+1}(\tau, u)=\int_{-\infty}^{\infty} x_{i}(t) x_{i}^{*}(t-\tau) e^{-j 2 \pi u t} d t
$$

10) Repeat steps (2)-(9) for a desired number of iterations or until an acceptable quality is reached 
11) Use the waveform associated with the highest quality ambiguity function

$$
x(t)=x_{j}(t)
$$

where

$$
j=\underset{i}{\arg \min } Q_{i}
$$

\section{EXAMPLES}

This section will walk through three examples showing the algorithm in action. In all examples, a waveform will be generated with a sampling frequency of $20 \mathrm{kHz}$ and 751 samples, yielding an ambiguity function size of 1500 by 1500 samples. The algorithm was run for 100 iterations. Note that the waveforms produced are baseband waveforms. In practice, the waveform will typically be upconverted to a carrier frequency before emission, where the passband waveform will have a related, but non-identical ambiguity function.

Table I summarizes the final numerical results for all examples. The first two examples use binary minimization functions, meaning that they are made up of regions where the desired ambiguity is either 0 or is completely arbitrary. These types of minimization functions do not seek to find an ambiguity function with a particular shape, but rather to simply minimize the function in certain regions. The third example uses a minimization function which has a particular shape which is desired. We found the minimax methods worked better for binary minimization functions similar to the first two examples and the least-squares methods worked better for shape-forming minimization functions similar to the third example. As such, only plots using the optimal method are shown in each respective example, but final numerical results are included for all cases.

\section{A. Example I}

Suppose that the areas in a ring around the origin are to be minimized. The main lobe will ideally be confined to the area inside of the annulus in a range of $-3.7<\tau<3.7 \mathrm{~ms}$, $-1<u<1 \mathrm{kHz}$. Outside of this region, the annulus to be minimized is confined to a region of $-11.25<\tau<$ $11.25 \mathrm{~ms},-3<u<3 \mathrm{kHz}$. Figures 2 through 4 display the minimizing function and the ambiguity function of the optimized waveform (2), the time and frequency magnitudes of the optimized waveform (3), and the change in minimax quality as the algorithm iterates (4). Numerical results can be found in Table I.

\section{B. Example II}

The second example will assume that some prior knowledge as to the relative range of interfering targets is available, but their velocity is unknown. We wish to minimize all Doppler within certain range regions, in this case $\pm 6.05<\tau<$ $\pm 11.3 \mathrm{~ms}$ and $\pm 22.55<\tau< \pm 30.05 \mathrm{~ms}$. Figures 5 through 7 display the minimizing function and the ambiguity function of the optimized waveform (5), the time and frequency magnitudes of the optimized waveform (6), and the change in minimax quality as the algorithm iterates (7).
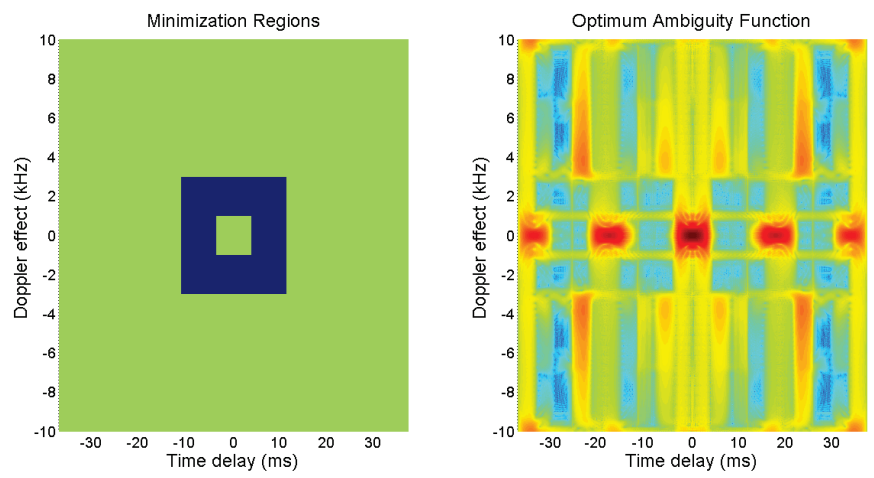

Fig. 2. (Ex. I) Left: $M(\tau, u)$. Right: magnitude (dB) of the optimized $\chi(\tau, u)$. The blue areas of $M(\tau, u)$ are the regions to be minimized. Generated using the minimax technique.
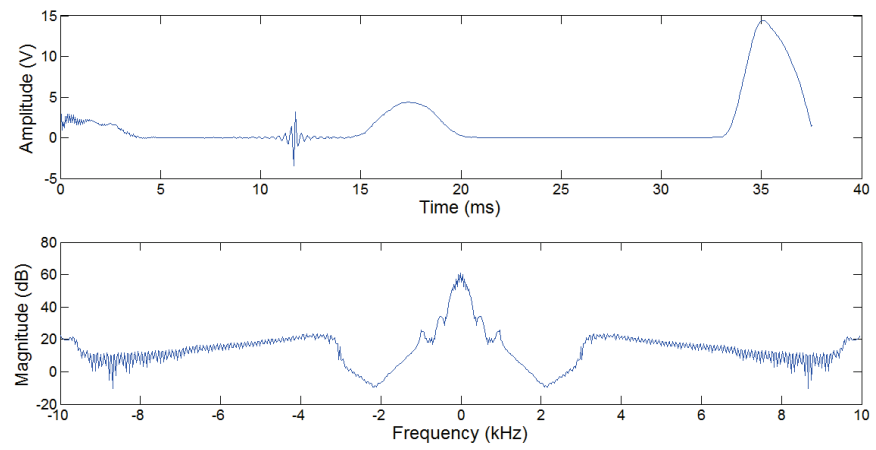

Fig. 3. (Ex. I) Top: the real part of the time domain signal $x(t)$ produced. Bottom: the magnitude of the spectrum of the signal produced.

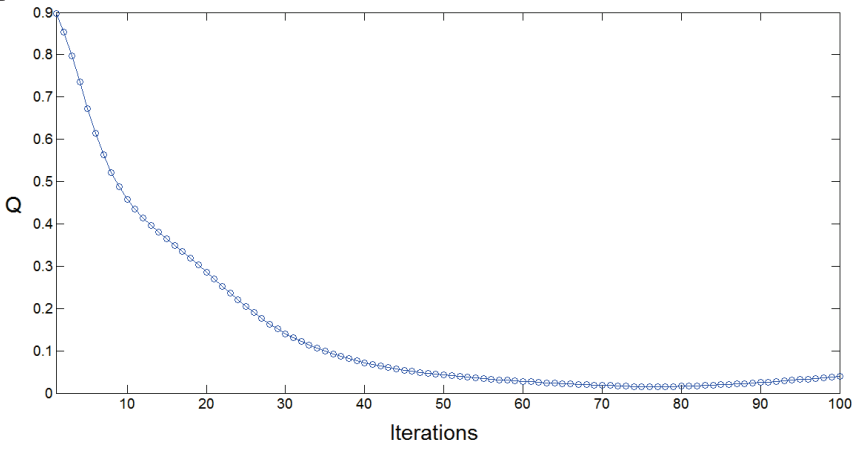

Fig. 4. (Ex. I) Minimax signal quality $Q_{i}$.

\section{Example III}

A slightly different application is shown in Example III, which displays the algorithm's robustness. Assume a situation in which a certain shape is desired, where the minimized area is relatively much greater than the area with high ambiguity. In this example, the area in which we seek to concentrate the ambiguity volume is within the diagonal line running across $M(\tau, u)$. The minimization function is given by

$$
M(\tau, u)=\left(1-\frac{\left|u \cdot \tau_{\max }-\tau \cdot u_{\max }\right|}{u_{\max } \cdot \tau_{\max }}\right)^{500}
$$

Figures 8 through 10 display the minimizing function and the ambiguity function of the optimized waveform (8), the time 

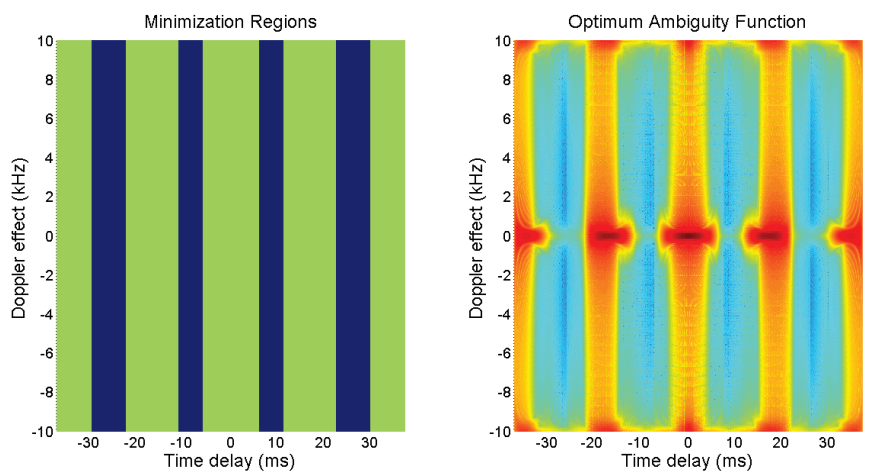

Fig. 5. (Ex. II) Left: $M(\tau, u)$. Right: magnitude $(\mathrm{dB})$ of $\chi(\tau, u)$. The blue areas of $M(\tau, u)$ are the regions to be minimized. Generated using the minimax technique.
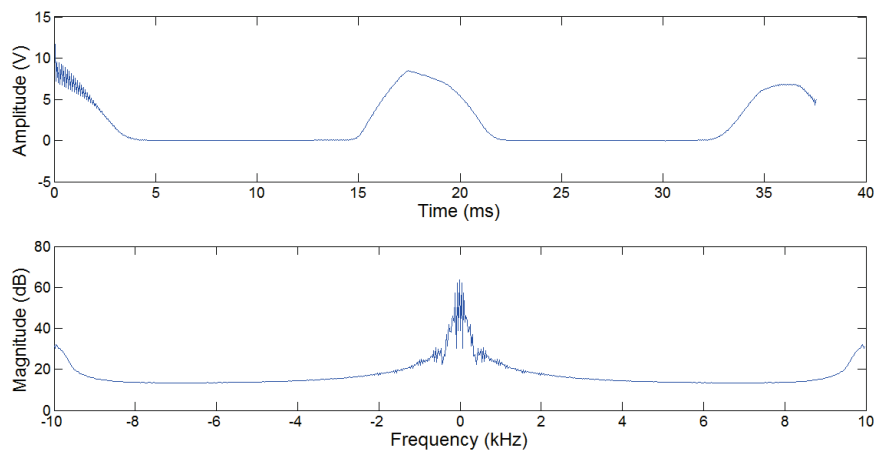

Fig. 6. (Ex. II) Top: the real part of the time domain signal $x(t)$ produced. Bottom: the magnitude of the spectrum of the signal produced.

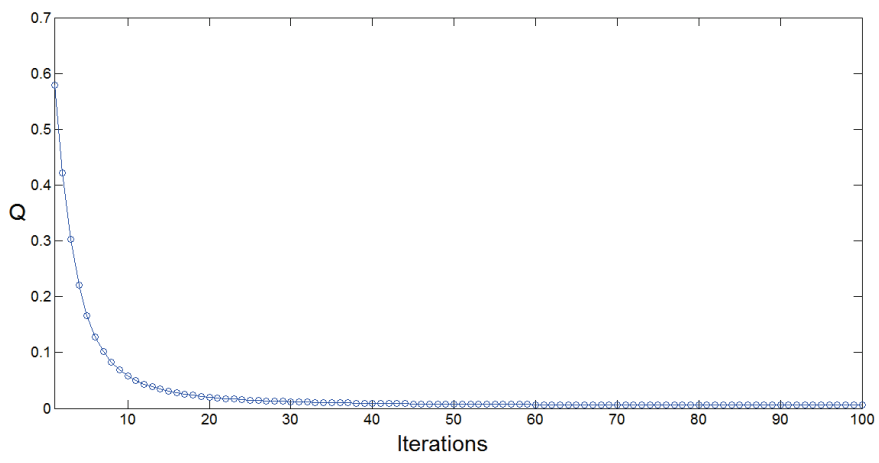

Fig. 7. (Ex. II) Minimax signal quality $Q_{i}$.

and frequency magnitudes of the optimized waveform (9), and the change in least-squares quality as the algorithm iterates (10).

TABLE I. Results from the Examples I and II

\begin{tabular}{llrrr}
\hline \multirow{2}{*}{ Optimum } & & & \multicolumn{2}{c}{ Quality Type } \\
\cline { 4 - 5 } & Weighting Style & Iteration & Least Squares & Minimax \\
\hline \multirow{2}{*}{ Example I } & Minimax & 77 & 0.0137 & 0.0141 \\
& Least-Squares & 65 & 0.0760 & 0.0274 \\
\multirow{2}{*}{ Example II } & Minimax & 100 & 0.00641 & 0.00804 \\
& Least-Squares & 75 & 0.00636 & 0.0957 \\
\multirow{2}{*}{ Example III } & Minimax & 6 & 1.124 & 0.465 \\
& Least-Squares & 16 & 0.651 & 0.847 \\
\hline
\end{tabular}
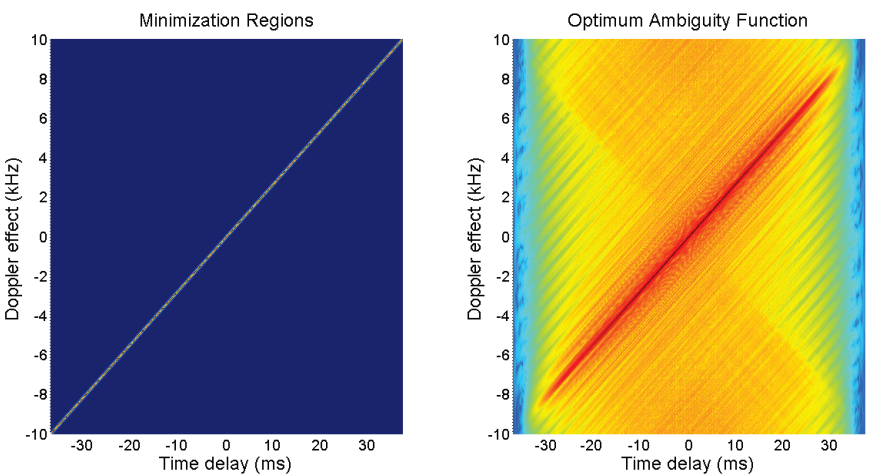

Fig. 8. (Ex. III) Left: $M(\tau, u)$. Right: magnitude $(\mathrm{dB})$ of $\chi(\tau, u)$. The diagonal line running through $M(\tau, u)$ is the area to be maximized. Generated using the least-squares technique.
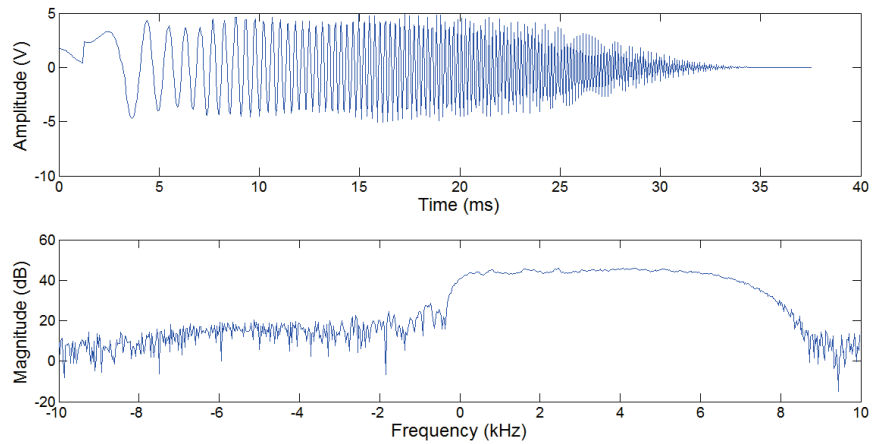

Fig. 9. (Ex. III) Top: the real part of the time domain signal $x(t)$ produced. Bottom: the magnitude $(\mathrm{dB})$ of the spectrum of the signal produced.

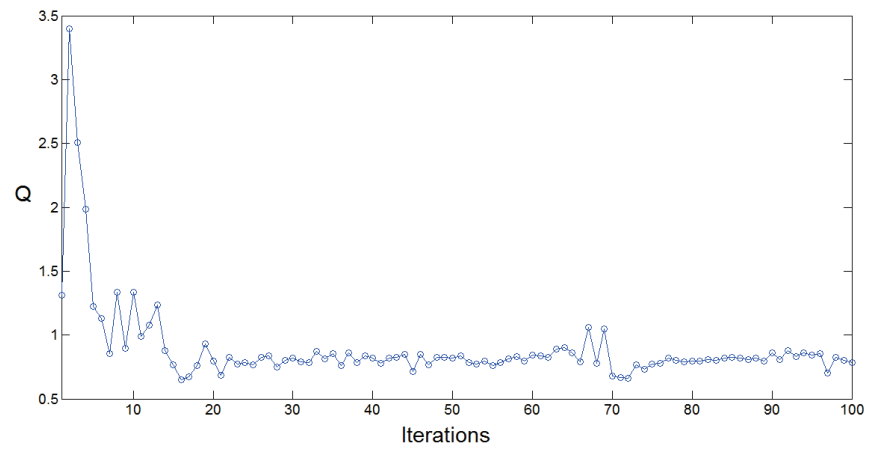

Fig. 10. (Ex. III) Least squares signal quality $Q_{i}$.

\section{CONCLUSION}

An algorithm was presented which uses a generalized projection onto convex sets approach to dynamically optimize a waveform based on minimization criteria in a desired ambiguity function. Examples of the algorithm successfully preforming optimization in realistic scenarios were shown. We found that the minimax weighting and distance functions were better used in minimization situations, while least squares weighting and integral distance function were better suited for shape-forming. Continuation of this research will include implementing the algorithm on a testbench in order to study the algorithm's effectiveness when non-linearitites introduced by a power amplifier are introduced. The results from this 
paper will be useful for waveform optimization in radar transmitters. We plan to expand our optimizations to include considerations of spectral compliance in the optimization, and to jointly opt imize the waveform with the nonlinear power amplifier.

\section{ACKNOWLEDGMENT}

This work has been funded under a grant from the National Science Foundation (Award Number ECCS-1343316). The authors would like to thank Keysight Technologies for costfree loan of the Advanced Design System simulation software.

\section{REFERENCES}

[1] Philip Mayne Woodward. Radar ambiguity analysis. Technical report, DTIC Document, 1967.

[2] Robert Price and Edward M Hofstetter. Bounds on the volume and height distributions of the ambiguity function. Information Theory, IEEE Transactions on, 11(2):207-214, 1965.

[3] John P Costas. A study of a class of detection waveforms having nearly ideal rangedoppler ambiguity properties. Proceedings of the IEEE, 72(8):996-1009, 1984.

[4] S Sussman. Least-square synthesis of radar ambiguity functions. Information Theory, IRE Transactions on, 8(3):246-254, 1962.

[5] Hao He, Petre Stoica, and Jian Li. On synthesizing cross ambiguity functions. In Acoustics, Speech and Signal Processing (ICASSP), 2011 IEEE International Conference on, pages 3536-3539. IEEE, 2011.

[6] Satyabrata Sen and Arye Nehorai. Adaptive design of ofdm radar signal with improved wideband ambiguity function. Signal Processing, IEEE Transactions on, 58(2):928-933, 2010.

[7] LG Gubin, BT Polyak, and EV Raik. The method of projections for finding the common point of convex sets. USSR Computational Mathematics and Mathematical Physics, 7(6):1-24, 1967.

[8] Heinz H Bauschke and Jonathan M Borwein. On projection algorithms for solving convex feasibility problems. SIAM review, 38(3):367-426, 1996.

[9] M REHA Civanlar and R Nobakht. Optimal pulse shape design using projections onto convex sets. In Acoustics, Speech, and Signal Processing, 1988. ICASSP-88., 1988 International Conference on, pages 1874-1877. IEEE, 1988.

[10] R Nobakht and M Reha Civanlar. Optimal pulse shape design for digital communication systems by projections onto convex sets. Communications, IEEE Transactions on, 43(12):2874, 1995

[11] Joel A Tropp, Inderjit S Dhillon, RW Heath, and Thomas Strohmer. Cdma signature sequences with low peak-to-average-power ratio via alternating projection. In Signals, Systems and Computers, 2004. Conference Record of the Thirty-Seventh Asilomar Conference on, volume 1, pages 475-479. IEEE, 2003.

[12] Robert J Marks. Handbook of Fourier analysis \& its applications. Oxford University Press London, 2009.

[13] Matthew Fellows, Charles Baylis, Lawrence Cohen, and RJ Marks. Radar waveform optimization to minimize spectral spreading and achieve target detection. In Wireless and Microwave Circuits and Systems (WMCS), 2013 Texas Symposium on, pages 1-4. IEEE, 2013. 